
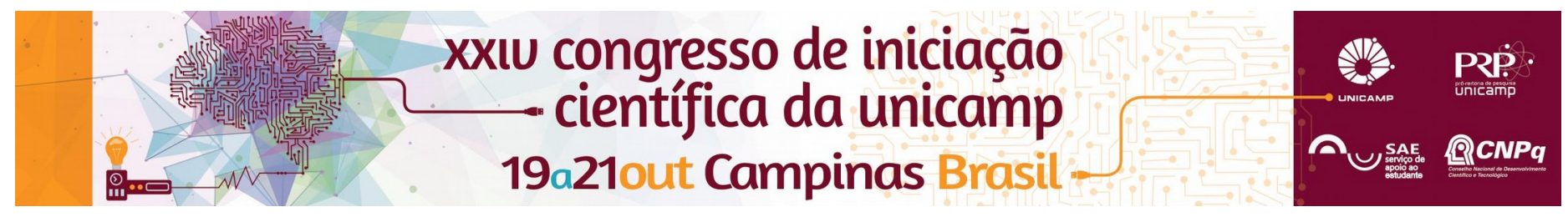

\title{
Exploring complex networks for data analysis
}

\section{Victor Roth Cardoso, André Santanchè}

\begin{abstract}
This work started as a proposal of complex networks investigation in Biology. Some findings in this initial topic conducted us to a more general study of the multiple facets of these networks including: data extraction of knowledge bases in network format; the generation of synthetic networks; analysis of data as networks with the proposal of new algorithms, including an original multilayer model. This model enables to distinguish the interaction of different aspects when simulating a given phenomenon as a network, which culminated in the construction of a tool for evaluating social models in networks. Since our multilayer model is the main contribution of this work, it is detailed in this document.
\end{abstract}

\section{Key words:}

Complex network, data analysis, social models

\section{Introduction}

One approach for modeling and simulating social networks involves the description of individuals through their characteristics, as well as the mechanism to transmit these characteristics between individuals. Based on this approach this work proposes a multilayer model to simulate network phenomena. We adapted and reproduced experimentally classic works, studying the produced modifications in our multilayer model.

\section{Results and Discussion}

This research started creating prototypes to reproduce experiments from the literature for three models $\mathbf{s}^{1,2,3}$, as well as their modification for a proposed multilayer model. The experiments required a network (topology) and a set of characteristics related to each network node.

The work focused on the experimental reproduction of the behavior of social models, involving the convergence on physical groups. It studies variations for a multilayer model, in which the network is segmented in many layers. Each layer represents the same individuals, however, there is a division of characteristics between the different layers, i.e. when using two layers half of the characteristics would be represented in each layer. We were investigating how people with specific characteristics in different social contexts, influenced by their similarity with another person, could change their characteristics along the time.

A novel approach for a multilayer model is proposed with the separation of the nodes' characteristics in distinct networks/layers, which can represent distinct contexts. We have adapted the existing models ${ }^{1,2,3}$, segmenting the comparison of similarity according to the available characteristics in the layer. However, if a characteristic would be transmitted, the whole range of characteristics is considered.

The experiments tried to simulate how individuals, inserted in different social contexts and influenced by the proximity to other individuals, can change from one state to another along the time. For example, a person that belongs to a social group of his job (one layer) could end up joining a football social group (another layer) due to this transmission of characteristics. Chart 1 presents the result of an analysis using a tool that we implemented. As can be seen, the number of physical groups tend to grow proportionally to the number of characteristics - which increases the potential diversity - and when we consider more than one context (layer) it changes the behavior.

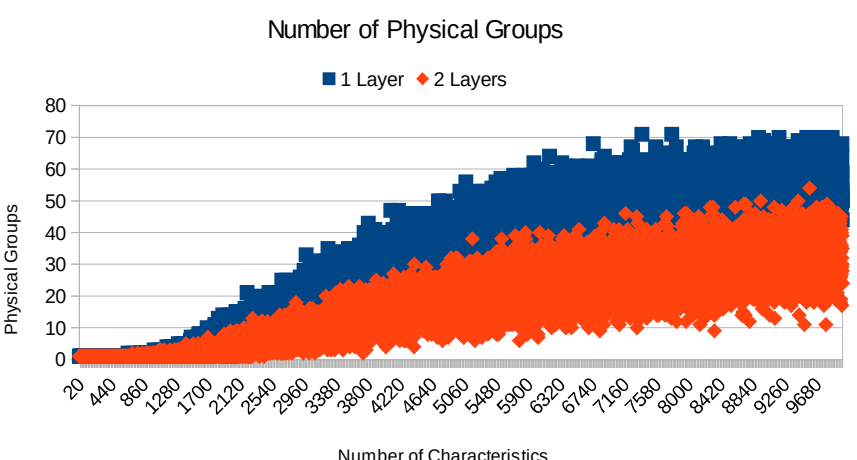

Chart 1. In red, the experiment was executed for two layers and in blue for one layer. It was based on Centola's algorithm².

\section{Conclusions}

The work on the models described here are available on $\mathrm{GitHub}^{4}$. They have been implemented using a tool that we created. This tool started reconstructing literature experiments and it has been transformed into a multipurpose tool.

\section{Acknowledgement}

This work is part of a join research developed with Kleber Andrade Oliveira and Felipe Maciel Cardoso. This work would not be possible without the support from the funding agencies ${ }^{5}$

\footnotetext{
${ }^{1}$ Axelrod, R. The Dissemination of Culture: A Model with Local Convergence and Global Polarization. Journal of Conflict Resolution. 1997, 203-226, 41(2). ${ }^{2}$ Centola, D., Gonzalez-Avella, J. C., Eguiluz, V. M., \& San Miguel, M. Homophily, Cultural Drift, and the Co-Evolution of Cultural Groups. Journal of Conflict Resolution. 2007, 905-929, 51(6).

${ }^{3}$ Klemm, K., Eguiluz, V. M., Toral, R., \& Miguel, M. S. Global culture: A noise induced transition in finite systems. Physics Review E. 2002, 67(4). ${ }^{4}$ Cardoso, Victor R. GitHub Codes.

https://www.github.com/labrax/socialtoolkit/. 2016.

${ }^{5}$ Work partially financed by FAPESP/Cepid in Computational Engineering and Sciences (2013/08293-7), the Microsoft Research FAPESP Virtual Institute (NavScales project), CNPq (MuZOO Project), FAPESP-PRONEX (eScience project), INCT in Web Science, and an individual grant from CNPq.
} 